\title{
Estimating Density and Effective Area Surveyed for American Woodcock
}

STEFANIE M. BERGH, ${ }^{1,2}$ Minnesota Cooperative Fish and Wildlife Research Unit, University of Minnesota, St. Paul, MN 55108, USA

DAVID E. ANDERSEN, U.S. Geological Survey, Minnesota Cooperative Fish and Wildlife Research Unit, University of Minnesota, St. Paul, MN 55108, USA

\begin{abstract}
The American Woodcock (Scolopax minor; hereafter, woodcock) Singing-ground Survey (SGS) is conducted annually during the woodcock breeding season, and survey points along survey routes are set 0.4 mile $(0.65 \mathrm{~km})$ apart to avoid counting individual birds from $>1$ listening location. The effective area surveyed (EAS) at a listening point is not known, and may vary as a function of land-cover type or other factors. To define the relationship describing distance between vocalizing woodcock and an observer and how cover types influence that relationship, we broadcast a recording of woodcock vocalizations in 2 land-cover types (forest and field) at varying distance. We evaluated the proportion of call broadcasts detected as a function of distance and fit regression curves to detection data to estimate a distance $\left(\mathrm{r}^{\star}\right)$ where the area above the curve at distances $\left\langle\mathrm{r}^{\star}\right.$ was equal to the area under the curve at distances $>\mathrm{r}^{\star}$, which allowed determination of the radius of an area where detection probability was effectively 1.o. This EAS had a radius $\left(\mathrm{r}^{\star}\right)$ of $198 \mathrm{~m}$ for forest, $384 \mathrm{~m}$ for field, and $309 \mathrm{~m}$ for both of these land-cover types combined, and an estimated size of 12.3 ha for forest, 46.3 ha for field, and 30.0 ha for both land-cover types combined. We used this information to estimate density of displaying male woodcock based on counts from the SGS in east-central Minnesota that incorporated variation in EAS, probability of detection, survey date, and survey route. Our density estimates (5.0 birds/10o ha in 2009 and 7.1 birds/100 ha in 2010) represent the highest density of singing male American woodcock yet reported, and indicated a substantive increase in density between years.
\end{abstract}

Proceedings of the American Woodcock Symposium 11: 193-199

KEY WORDS: American woodcock, detection distance, effective area surveyed, Minnesota, Scolopax minor

The American woodcock (Scolopax minor; hereafter, woodcock) Singing-ground Survey (SGS), coordinated by the U. S. Fish and Wildlife Service (FWS) and the Canadian Wildlife Service, is conducted during the woodcock spring breeding season. This roadside survey is conducted in the evening when males make a distinctive vocalization called peenting as part of their breeding display (Keppie and Whiting 1994). Each male occupies its own open area called a singing ground where it peents to attract female woodcock and advertise occupancy to conspecific males. Woodcock use a variety of openings (natural openings, clearcuts, agricultural fields, etc.) for this display.
The SGS has been conducted annually throughout the primary woodcock breeding range in the eastern U.S. and adjacent southern Canada since 1968, and is used as an index of abundance and population trend. There are approximately 1,500 SGS routes that are 3.6 miles $(5.8 \mathrm{~km})$ in length and have 10 listening points spaced 0.4 miles $(0.65 \mathrm{~km})$ apart (Seamans and Rau 2017). Observers begin surveys shortly after sunset and record the number of woodcock heard peenting at each listening point during a 2-min listening period. From 1968 to 2017, the number of singing male woodcock counted per route on the SGS has declined (Seamans and Rau 2017) in both the Eastern and

1 email: Stefanie.Bergh@dfw.wa.gov

2 current address: Washington Department of Fish and Wildlife, P.O. Box 484,

White Salmon, WA 98672, USA

https://doi.org/10.24926/AWS.0125 CC BY 4.0 
Central Management Regions, with a more recent period (2007-2017) of stability in the Central Management Region and across the breeding distribution as a whole. Concerns about this decline have led to harvest restrictions (summarized in Seamans and Rau 2017), a woodcock conservation plan (Kelley et al. 2008), and a need to better understand how counts of woodcock on the SGS are related to woodcock abundance and population trends.

As currently implemented and analyzed, the SGS provides an estimate of males per route rather than males per unit area. The SGS protocol is designed to maximize detection probability of singing male woodcock by defining the conditions under which surveys are conducted, and by controlling for several factors that influence detection probability (Bergh 2011). However, detection probability on SGS surveys was not previously known, and by accounting for factors that influence detection, evaluation of trends in woodcock abundance could be improved. One factor that likely influences detection is the distance from a bird to the observer. Recent experiments (see Simons et al. 2007, McClintock et al. 2010) documented that detection probability decreases with distance, especially in the presence of ambient noise. Increasing distance can lead either to misidentification or to false-positive or false-negative detections. Therefore, it is important to estimate the proportion of birds detected as a function of distance and to understand factors that influence detection.

The farthest distance at which an observer can detect various songbirds has been investigated empirically by broadcasting recordings of calls. For example, Emlen and DeJong (1981) introduced the idea of detection threshold distances (DTDs), which they defined as the distance at which a bird song becomes inaudible in a natural setting. These DTDs could then be translated to detection areas (DAs) and applied to counts of singing birds along a survey route. A DTD would be determined for a particular species and cover type, eliminating the need for an observer to estimate the distances to singing birds during a survey. However, the use of a single threshold number was questioned by Wolf et al. (1995) when they fit a theoretical function to describe the relationship between distance and detectability. The value of interest (D50) described the distance where one-half of the birds of a given species were audible during a point count, so that the probability of hearing a bird within that distance was equal to that of missing a bird beyond that distance. These studies provided crude estimates of the farthest distance at which an observer could detect various vocalizing songbirds in a forest and provided a basis for evaluating detection distance on the SGS.

The SGS protocol was developed partly on evaluations conducted by Duke (1966) of factors related to woodcock peenting. Duke (1966) estimated the distance at which peenting woodcock could be heard, and concluded that none were detected beyond 257 yards $(235 \mathrm{~m})$. He recommended that the FWS maintain a 0.4 -mile $(0.65-\mathrm{km})$ interval between stops on SGS routes to avoid counting individual birds from $>1$ listening location. This resulted in a 0.2 -mile (approximately 330-m) radius around each listening point and an estimated effective area surveyed (EAS) of 34.2 ha (assuming all woodcock peenting were detected). Alternatively, Gregg (1984) assumed that an SGS observer would hear all peenting male woodcock within a 220-yard $(201-\mathrm{m})$ radius of a listening point for an EAS of 12.7 ha. However, after calculating a very low density of woodcock along Wisconsin SGS routes with this presumed area surveyed, Gregg (1984) concluded that the estimate of the area within which woodcock were detected along routes may have been too large. More recently, Kelley et al. (2008) suggested a listening-point radius of $250 \mathrm{~m}$, which amounts to an EAS of 19.6 ha. These estimates of the area within which woodcock are detected at SGS survey locations vary widely, with the largest estimate from the current SGS protocol.

A reliable estimate of the EAS can be used to estimate density of male singing woodcock. Several studies have attempted to estimate density without having directly estimated the EAS for an SGS listening point. Gregg (1984) compared breeding woodcock densities in the literature based on singing-grounds/10o acres (40.7 ha) in the Midwest and northeastern United States. $\mathrm{He}$ assumed an occupied singing-ground was equivalent to 1 singing male woodcock. His density estimates ranged from 0.7 to 4.2 singing-grounds/10o acres (1.7 to 10.4 singing-grounds/10o ha). Dwyer et al. (1988) estimated woodcock density on Moosehorn National Wildlife Refuge in Maine by counting all singing males in a study area of known size and assuming that no birds were missed. Their density estimates ranged from 1.3 to 2.2 singing males/10o ha over a 10-yr study period. In the American Woodcock Conservation Plan, density estimates were made for all the counties within the breeding distribution of woodcock. Kelley et al. (2008) used a radius of $250 \mathrm{~m}$, but this estimate of detection distance was not based on empirical data. Kelley et al. (2008) were interested in comparing woodcock populations from 1970-1975 to those of 2000-2004 and used the average number of singing males per route during those periods. They represented counties by their official SGS routes, and resulting density estimates for Pine County, Minnesota, USA (the location of our study), were 0.88 singing males/10o ha for 1970-1975 and 0.75 singing males/10o ha for 2000-2004.

To date, there has not been a thorough evaluation of the relationship between distance and detection of peenting woodcock at a listening point on the SGS, which precludes using SGS data to estimate woodcock density. Furthermore, many factors likely influence woodcock detection 
probability, such as land-cover type (e.g., forest, agriculture, urban, etc.), environmental conditions under which surveys are conducted, and abilities of observers. Therefore, our objectives were to estimate the EAS at SGS listening points with respect to land-cover type using both open field and forested locations. We predicted that land-cover type would affect the probability of detecting a peenting woodcock as a function of distance. We also incorporated EAS and detection probability with SGS counts to derive estimates of male woodcock density in Pine County, Minnesota, USA.

\section{Study Area}

We derived distance-detection relationships from trials we conducted in open field and forested land-cover types in Pine County, Minnesota, in 2009 and 2010. Pine County is located in east-central Minnesota, and our study sites were located near the town of Finlayson (46.203 N, -92.956 W). Pine County is situated in the Mille Lacs Uplands subsection under the Ecological Classification System hierarchy (Minnesota DNR 2006). This subsection is characterized by drumlin ridges with depressions between the ridges containing peatlands with shallow organic material. There are extensive wetlands in the area with total annual precipitation of about $75 \mathrm{~cm}$. Large areas in eastern Pine County are heavily forested. The county is dominated by aspenbirch (Populus spp.-Betula spp.) forest with small areas of jack-white-red pine (Pinus spp.) forests. Land ownership in the Mille Lacs Uplands subsection is $17.7 \%$ public and $82.2 \%$ private, and land use is approximately $40 \%$ forest, $24 \%$ row crop, $17 \%$ wetland-open, $13 \%$ pasture, and $6 \%$ water (Minnesota DNR 2006).

\section{Methods}

\section{DATA COLLECTION}

We conducted call-broadcast trials at 9 sites, 4 that we categorized as forest and 5 that we categorized as open field. Forest sites were topographically flat and vegetated by mixed pine forest, mature aspen forest interspersed with alders (Alnus spp.) in a wet area, mixed pine forest and pine plantation, and mixed pine forest with birch, aspen, and a willow (Salix spp.) and alder wet area. Open field sites were also topographically flat; 2 were horse pasture, 2 were hayfields, and 1 was a restored native prairie. Two of the forest sites were public land, whereas the remaining sites were located on private land.

To estimate the farthest distance at which we could detect peenting woodcock, we broadcasted a recording of a woodcock peent through speakers at a sound level between 70 and 80 decibels (field trials and e.g., Brackenbury 1979, Simons et al. 2007). While 1 observer stood blindfolded on a road, another individual held a game caller (FOXPRO FX, FOXPRO Inc., Lewistown, PA) at a distance unknown to the observer and either played or did not play the recording. Broadcast distances were set at $50-\mathrm{m}$ increments between 100 and $450 \mathrm{~m}$ (open field) or $100 \mathrm{~m}$ and $300 \mathrm{~m}$ (forest) based on preliminary assessments. The observer listened for $2 \mathrm{~min}$ and recorded whether they heard peenting. We recorded wind speed, precipitation, and level of ambient noise during the trial following the official SGS protocol (e.g., we did not conduct trials in heavy wind or precipitation). We conducted broadcast trials primarily in the hours during and after sunrise (06:00-09:00) to simulate the conditions during which the official SGS is conducted. We did not conduct trials during the hours around sunset because observers were conducting woodcock surveys during that period as part of a companion study. We conducted trials in April and May of 2009 and 2010 over multiple days and sites in the 2 land-cover types (open forest and open field) to estimate detection distance and to compare detection distance between land-cover types. Observers had their hearing professionally evaluated prior to conducting trials and were trained to listen for woodcock.

\section{DATA ANALYSIS}

We calculated the proportion of peent broadcasts detected at each distance and in each land-cover type (Fig. 1). Based on the proportion of broadcasts detected and with the assumption that all broadcasts at $\mathrm{o} \mathrm{m}$ from the observer would be detected, we used program R ( R Development Core Team 2010) to analyze 4 different curves (half normal, inverse normal, negative exponential, and logistic) to determine the detection curve with the best fit. We ranked these 4 a priori candidate models using Akaike's Information Criterion adjusted for sample size $\left(\mathrm{AIC}_{c}\right)$ for the field and forest land-cover types to identify the model best sup-

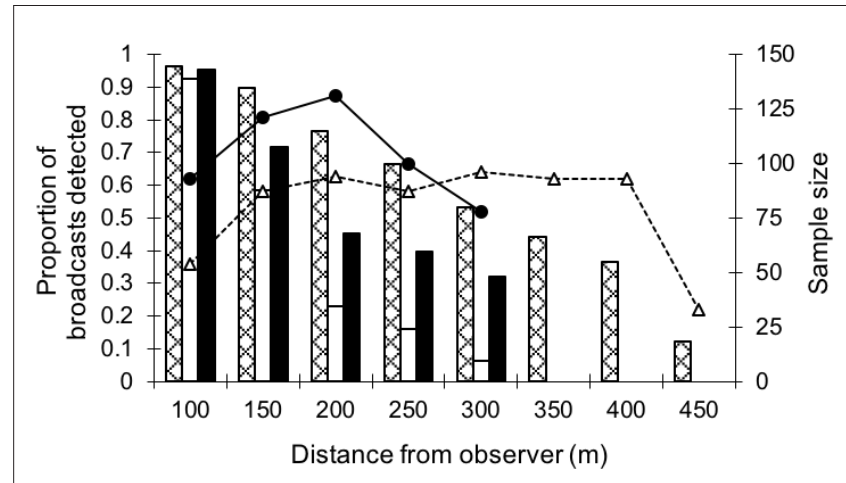

Figure 1. Proportion of broadcasts of American woodcock "peents" detected at increasing distance (m) from an observer in open field (crosshatch), forest (white), and overall (black) land-cover types in Pine County, Minnesota, 2009-2010. Lines show sample sizes for open field (dotted) and forest (solid) land-cover types at each distance. 
ported by the data (Burnham and Anderson 2002). We then used the best-supported detection curve (half-normal) to estimate the EAS, following the procedure outlined in Roberson et al. (2005) where probability of detection is a function of distance. In that procedure, the ideal probability of detection $\left(P_{i}\right)$ is equal to 1 out to a given distance $(\mathrm{x}, \mathrm{y})$ from the source of the broadcast $(\mathrm{o}, \mathrm{o})$ and zero beyond that distance. The next step is to set the double integral of $P_{i}$ equal to that of $P_{t}$, the probability of detection as a function of distance based on the data. We then solved for $\mathrm{r}^{\star}$, the radius of the EAS (and the $\mathrm{x}$ coordinate on the detection curve), which is the distance at which the area above the probability of detection curve at distances $<\mathrm{r}^{\star}$ equals the area under the curve at distances $>\mathrm{r}^{\star}$. For a half-normal curve the $\mathrm{r}^{\star}$ can be calculated by

$$
\mathrm{r}^{\star}=2\left(\sqrt{ } \int \mathrm{Pt}(\mathrm{r}) \mathrm{dr}\right)
$$

where $P_{t}$ is the probability of detection at distance $t$ and $r$ is the radius from the location where the peent call was broadcast. Following integration, the equation for $\mathrm{r}^{\star}$ was reduced to

$$
r^{*}=\sqrt{2} \sigma^{2}
$$

where $\sigma$ is the standard deviation from a half-normal distribution. We used this radius to determine the effective area surveyed:

$$
\mathrm{EAS}=\pi\left(\mathrm{r}^{\star}\right)^{2}
$$

We calculated a 95\% bootstrap confidence interval for $\mathrm{r}^{\star}$ with 1,000 bootstrap samples using program $\mathrm{R}$ to assess uncertainty in the EAS. We repeated this procedure for forest, open field, and forest and open field combined land-cover types.

We then used bootstrapping to estimate density of peenting male woodcock in Pine County. We estimated density for each year (2009 and 2010) using our estimates of EAS, detection probability $(p ; 0.625$ pooled from the 2009 and 2010 estimates of detection probability, Bergh 2011), and observed counts of woodcock on each of the county's 8 routes during the official SGS window for central Minnesota (25 April-20 May). We conducted 2,000 simulations, randomly selecting 8 routes (with replacement, i.e. bootstrapping the routes), the same number of dates for each year-route combination (i.e., bootstrapping the dates), EAS based on cover type and its estimated mean and variance (parametric bootstrapping the EAS), and detection probability $(p)$ based on its estimated mean and variance (parametric bootstrapping $p$ ). When sampling for EAS, we used a shifted gamma random variable by sampling from a gamma distribution with a given shape, then multiplying by a given scale, then adding a given shift to match the mean $(m), 2.5 \%$ quantile $(a)$, and $97.5 \%$ quantile $(b)$ of the estimated distribution of EAS in each of the 3 cover types (described above). To determine the shape of the gamma distribution, we computed the ratio:

$$
(m-a) /(b-a)
$$

and then used that ratio to define a gamma distribution to achieve the same ratio. To determine the scale, we computed:

$$
(b-a)
$$

and divided that difference by the corresponding quantity for a standard gamma with the derived shape. Finally, the shift parameter is $m$ minus the product of shape and scale. When sampling for $p$, we used a normal distribution with mean 0.625 and variance 0.0176 (Bergh 2011).

For each replicate in the simulation $(n=2,000)$, we therefore allowed for variation in EAS, $p$, survey date (within the SGS window), and route and estimated density by summing the adjusted number of woodcock observations and dividing by the sum of estimated EAS across all points on all routes. We assigned the appropriate EAS to each point on each route from U.S. Farm Service Agency aerial photos and ground observations (Bergh 2011). We used the $2.5 \%$ and $97.5 \%$ quantiles of the distribution of replicates in the simulation to represent $95 \%$ confidence intervals around point estimates of woodcock density (birds/10o ha) for 2009 and 2010 in Pine County.

\section{Results}

\section{EFFECTIVE AREA SURVEYED}

We conducted a total of 1,160 woodcock broadcast trials at 5 distances in the forest land-cover type and 8 distances in the open field land-cover type, for an average of approximately 90 trials per distance in each land-cover type. We conducted trials over 19 days in 2009 and 25 days in 2010. The percentage of broadcasts detected ranged from $96.3 \%$ and $92.5 \%$ at $100 \mathrm{~m}$ in the open field and forest land-cover types, respectively, to $12.1 \%$ at $450 \mathrm{~m}$ in the open field landcover type and $6.4 \%$ at $300 \mathrm{~m}$ in the forest land-cover type. Detection probability decreased less rapidly as a function of distance in the open field land-cover type than in the forest land-cover type (Fig. 1).

The best-fit detection curve for all 3 datasets (forest, open field, both land-cover types combined) was half-normal (Table 1, Fig. 2). No other models received substantial support; therefore we used the parameter estimates from the half-normal curve defined by our data to calculate the EAS. The EAS radius $\left(\mathrm{r}^{\star}\right)$ was $198 \mathrm{~m}(95 \%$ bootstrap CI $=$ $174-231 \mathrm{~m}$ ) for the forest land-cover type, $384 \mathrm{~m}$ (95\% bootstrap CI $=321-440 \mathrm{~m}$ ) for the open field land-cover type, and $309 \mathrm{~m}$ ( $95 \%$ bootstrap CI $=273-372 \mathrm{~m}$ ) for both landcover types combined. The EAS for SGS listening points in Pine County was 12.3 ha (95\% bootstrap CI $=9.46-16.8$ ) for the forest land-cover type, 46.3 ha (95\% bootstrap 
$\mathrm{CI}=32.4-60.8)$ for the open field land-cover type, and 30.0 ha $(95 \%$ bootstrap CI $=23.4-43.4$ ) for both land-cover types combined.

\section{DENSITY}

Mean density of singing male woodcock was 5.0 birds/100 ha (95\% confidence interval $2.2-9.6)$ in 2009 and 7.1 birds/10o ha (95\% confidence interval 3.6-12.6) in 2010, suggesting an increase in abundance between years. Bootstrapping $p$ made relatively little difference in the lower bound of our confidence intervals, but increased the upper bound (up to approximately 2 birds/10o ha). Bootstrapping route lowered the lower bound of our confidence intervals and increased the upper bound slightly (up to approximately 1-2 birds/10o ha). Bootstrapping EAS and date had relatively little influence on confidence interval bounds derived via simulations.

\section{Discussion}

\section{EFFECTIVE AREA SURVEYED}

We estimated the EAS for American woodcock in open field and forest land-cover types in east-central Minnesota based on call broadcast trials conducted under a variety of conditions within the limitations of the SGS protocol, in relatively flat terrain, and during the hours around sunrise. We conducted trials over many days in a variety of environmental conditions, wind speeds and directions, ambient noise levels, and precipitation. Therefore, our estimates of the EAS should be considered averages over the conditions under which SGSs are conducted. Although

Table 1. Akaike's Information Criterion adjusted for sample size $\left(\mathrm{AIC}_{\mathrm{c}}\right)$, the difference in $\mathrm{AIC}_{\mathrm{c}}\left(\Delta \mathrm{AIC}_{\mathrm{c}}\right), \mathrm{AIC}_{\mathrm{c}}$ model weights $\left(\omega_{i}\right)$, and number of parameters in the model $(k)$ from the best-supported model for 4 a priori models of the relationship between American woodcock detection and distance in a forest, open field, and both land-cover types combined in Pine County, Minnesota, 2009-2010.

\begin{tabular}{llcccc}
\hline $\begin{array}{l}\text { Land-cover } \\
\text { type }\end{array}$ & Model & $\mathrm{AIC}_{\mathfrak{c}}$ & $\Delta \mathrm{AIC}_{\mathbf{c}}$ & $\boldsymbol{\omega}_{\boldsymbol{i}}$ & $\boldsymbol{k}$ \\
\hline Forest & Half Normal & 0.6230 & 0.000 & 0.9591 & 2 \\
& Negative Exponential & 6.942 & 6.319 & 0.0407 & 2 \\
& Logistic & 19.17 & 18.55 & 0.0001 & 3 \\
& Inverse Normal & 19.63 & 19.01 & 0.0001 & 3 \\
\hline Open & Half Normal & -8.513 & 0.000 & 0.9508 & 2 \\
field & Negative Exponential & -1.357 & 7.156 & 0.0266 & 3 \\
& Logistic & -0.8730 & 7.640 & 0.0208 & 3 \\
& Inverse Normal & 3.984 & 12.497 & 0.0018 & 2 \\
\hline Both & Half Normal & -6.477 & 0.000 & 0.6058 & 2 \\
combined & Negative Exponential & -4.707 & 1.770 & 0.2500 & 2 \\
& Logistic & -2.276 & 4.201 & 0.0742 & 3 \\
& Inverse Normal & -2.160 & 4.317 & 0.0700 & 3 \\
\hline
\end{tabular}

these trials were conducted in the hours around sunrise, instead of around sunset (as during the SGS), environmental conditions around sunrise are similar to those around sunset, and male woodcock display at both dusk and dawn (Sheldon 1967). Therefore, we conducted our trials around sunrise in conditions nearly identical to those around sunset, in terms of factors that influence detection of peenting woodcock.

The EAS in the open field land-cover type was greater than that in the forest land-cover type, likely because of sound attenuation in forest vegetation (Wiley and Richards 1982). Our estimate of EAS radius across land-cover types (field and forest combined) was $309 \mathrm{~m}$, which is similar to previous estimates of $201 \mathrm{~m}, 235 \mathrm{~m}, 250 \mathrm{~m}$, and 330 m (Gregg 1984, Duke 1966, Kelley et al. 2008, SGS protocol, respectively). However, only Duke's (1966) estimate was determined based on empirical data-the farthest distance he and others could hear 3 known singing males in 28 trials. Our detection distances were considerably farther than the $235 \mathrm{~m}$ reported by Duke (1966), especially in the field land-cover type. We do not know why our distances were farther than those reported by Duke (1966), but suspect detection distance is likely related to differences in land-cover type, observer's hearing abilities, and our more extensive and controlled testing protocol. These results also suggest that spatial or temporal comparisons of counts that do not account for detection probability may need to be made with caution. When combining data from both land-cover types, our estimate of the EAS was 30.0 ha, which extrapolates to a total of 300 ha effectively surveyed on a single SGS route (with 10 listening points).

\section{DENSITY}

The density estimates we derived for male singing woodcock in Pine County ( 5.0 birds/100 ha in 2009 and 7.1 birds/10o ha in 2010) are considerably greater than those previously estimated in other locations. The highest estimated density reported in the published literature was 2.2 birds $/ 100$ ha at Moosehorn National Wildlife Refuge (NWR) in Maine (Dwyer et al. 1988). The study area on Moosehorn NWR had high quality woodcock habitat that had recently undergone management specifically to benefit woodcock prior to the study (Dwyer et al. 1988), whereas our study was located in a mix of public and private lands that were not managed for woodcock. Estimated density for woodcock in Pine County presented in the American Woodcock Conservation Plan (Kelley et al. 2008) was also much lower than our estimates, indicating the potential impor- 


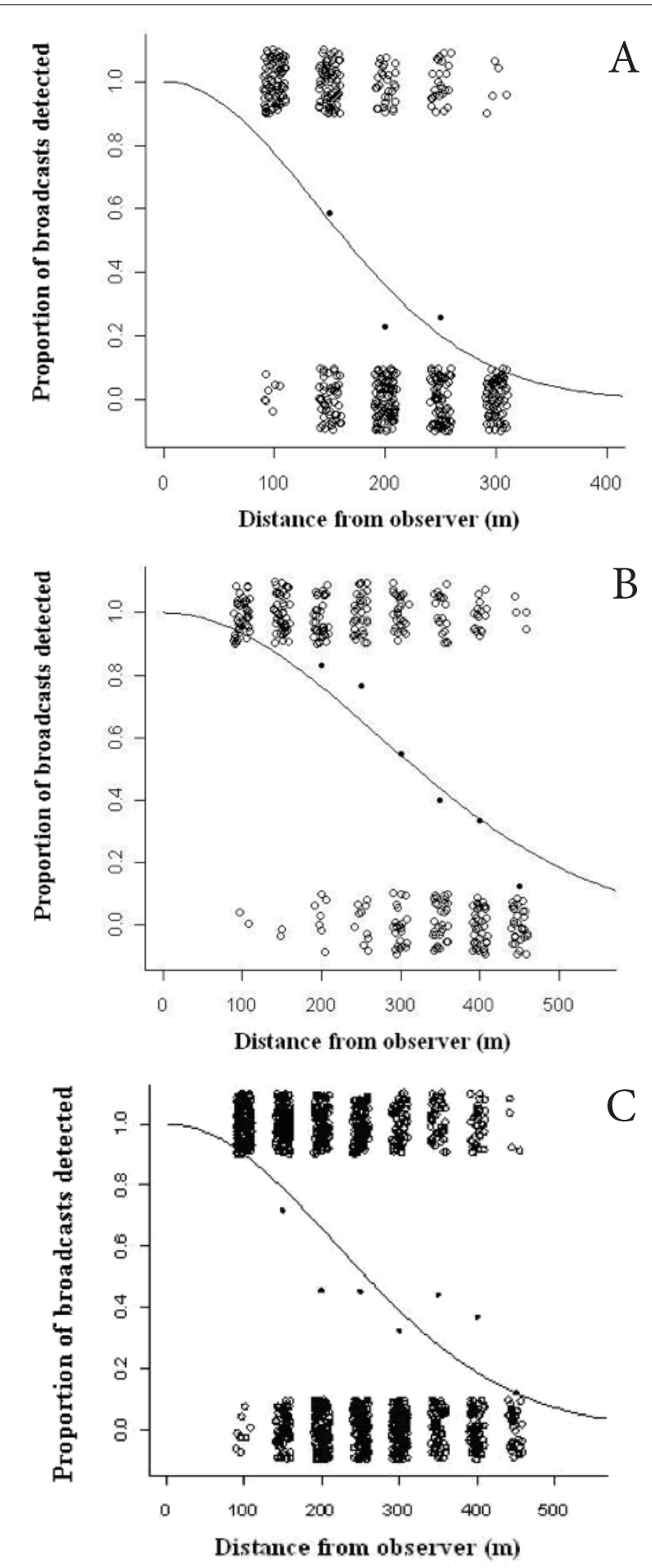

Figure 2. Proportion of broadcasts of American woodcock "peents" detected at increasing distance (m) from an observer in open field (crosshatch), forest (white), and overall (black) land-cover types in Pine County, Minnesota, 2009-2010. Lines show sample sizes for open field (dotted) and forest (solid) land-cover types at each distance. tance of incorporating detection probability into density estimates. In addition, raw counts and density estimates indicated a $39-42 \%$ increase in woodcock abundance on our routes from 2009 to 2010, suggesting that estimated abundance can vary substantively between years.

\section{Management Implications}

Based on our estimates of EAS in forested and open field land-cover types in east-central Minnesota, the $330 \mathrm{~m}$ radius currently used for SGS points appears adequate to ensure that woodcock are not counted on $>1$ survey point, unless consecutive survey points are completely surrounded by flat, open field. In that case the same bird has the potential to be counted at consecutive survey points, which violates the assumption of independent survey points. Recording the cardinal direction and approximate distance to a peenting woodcock in this situation might prevent an observer from counting the same bird twice. Not counting uncertain detections (i.e., birds heard faintly that are likely beyond reliable detection distance) will increase confidence in (1) reducing double counting of the same bird from consecutive points and (2) counting birds only within the EAS. In forested land-cover types, in contrast, observers likely would not detect woodcock beyond $198 \mathrm{~m}$, suggesting that one must consider land-cover type when comparing counts between locations.

Our estimates for the EAS at an SGS point can be used to calculate density of singing male woodcock. With technology such as remote sensing, SGS points may be easily classified into the 3 general land-cover categories we used in our study. The unique EAS estimates for each SGS route could also be evaluated periodically to account for changes in land cover. Our results also highlight the importance of accounting for detection probability and EAS on woodcock surveys at the scale of a county in east-central Minnesota. Detection probability and EAS are likely different in different landscapes, and may need to be assessed more broadly to assess how topography and other forest cover types are related to detection probability and EAS.

\section{Acknowledgments}

This project was funded by the U.S. Fish and Wildlife Service Webless Migratory Game Bird Research Program. J. R. Kelley assisted in generating support for this project. D. Prokop and the Finlayson-Giese Sportsmen's Club provided housing and support during field work. We are grateful for the hard work of field technicians J. A. Fritz, B. J. Oberschmid, N. R. Bieber, and J. C. Bietka. Helpful advice and comments were provided by T. R. Cooper, R. J. Gutiérrez, and D. H. Johnson. Statistical advice and help with program $\mathrm{R}$ were provided by the Statistics Department at the University of Minnesota, especially by G. W. Oehlert, who also conducted the density simulations. We conducted this study under protocol no. 0801A24506 of 
the University of Minnesota Animal Care and Use Committee. Use of trade names does not imply endorsement by either the U.S. Government or the University of Minnesota.

\section{Literature Cited}

Bergh, S.M. 2011. Factors influencing detection of American woodcock during Singing-ground surveys.M.S. Thesis, University of Minnesota, St. Paul, Minnesota, USA.

Brackenbury, J.H. 1979. Power capabilities of the avian sound-producing system. Journal of Experimental Biology 78:163-166.

Burnham, K.P., and D.R. Anderson. 2002. Model selection and inference: a practical information-theoretic approach. 2nd edition. Spring-Verlag, New York, NY.

Duke, G.E. 1966. Reliability of censuses of singing male woodcock. Journal of Wildlife Management 30:697-707.

Dwyer, T. J, G.F. Sepik, E.L. Derleth, and D.G. McAuley. 1988. Demographic characteristics of a Maine woodcock population and effects of habitat management. U.S. Fish and Wildlife Service, Fish and Wildlife Research 4, Washington, D.C., USA.

Emlen, J.T., and M.J. DeJong. 1981. The application of song detection threshold distance to census operations. Pages $346-352$ in C.J. Ralph and J.M. Scott (eds). Estimating numbers of terrestrial birds. Allen Press, Lawrence, Kansas, USA.

Gregg, L.E. 1984. Population ecology of woodcock in Wisconsin. Technical Bulletin 144. Wisconsin Department of Natural Resources, Madison, Wisconsin, USA.

Kelley, J.R., S.J. Williamson, and T.R. Cooper (eds.) 2008. American woodcock conservation plan: a summary of and recommendations for woodcock conservation in North America. Wildlife Management Institute, Washington, D.C., USA.

Keppie, D.M., and R.M. Whiting, Jr. 1994. American woodcock (Scolopax minor). Account 100 in A. Poole and F. Gill (eds). The birds of North America. Academy of Natural Sciences, Philadel- phia, Pennsylvania, and The American Ornithologists' Union, Washington, D.C., USA.

McClintock, B.T., L.L. Bailey, K.H. Pollock, and T.R. Simons. 2010. Experimental investigation of observation error in anuran call surveys. Journal of Wildlife Management 74:1882-1893.

Minnesota Department of Natural Resources. 2006. Tomorrow's habitat for the wild and rare: an action plan for Minnesota wildlife. Comprehensive Wildlife Conservation Strategy. Division of Ecological Services, Minnesota Department of Natural Resources. St. Paul, Minnesota, USA.

R Development Core Team. 2010. R: A language and environment for statistical computing, version 2.11.1. R Foundation for Statistical Computing, Vienna, Austria.

Roberson, A. M, D.E. Andersen, and P. L Kennedy. 2005. Do breeding phase and detection distance influence the effective area surveyed for northern goshawks? Journal of Wildlife Management 69:1240-1250.

Seamans, M.E. and R. D Rau. 2017. American woodcock population status, 2017.U.S Fish and Wildlife Service. Laurel, Maryland, USA.

Sheldon, W.G. 1967. The book of the American woodcock. University of Massachusetts Press, Amherst, MA.

Simons, T.R., M.W. Alldredge, K.H. Pollock, and J.M. Wettroth. 2007. Experimental analysis of the auditory detection process on avian point counts. Auk 124:986-999.

Wiley, R.H., and D.G. Richards. 1982. Adaptations for acoustic communication in birds: sound transmission and signal detection. Pages 132-182 in D.E. Kroodsma and E.H. Miller (eds). Acoustic communication in birds 1. Academic Press, New York, NY.

Wolf, A.T., R.W. Howe, and G.J. Davis. 1995. Detectability of forest birds from stationary points in northern Wisconsin Pages 19-23 in J. Ralph, J.R. Sauer, and S. Droege (eds). Monitoring bird populations by point counts. USDA Forest Service General Technical Report PSW-GTR-149, Pacific Southwest Research Station, Albany, California, USA. 